\title{
National Collaborative for Bio-Preparedness
}

\author{
Meredith Arasaratnam*1,2, David Potenziani ${ }^{1,2}$, Marc Hoit ${ }^{3,1}$, Colleen Jenkins ${ }^{4,1}$ and \\ Charles Cairns ${ }^{2,1}$
}

${ }^{1}$ National Collaborative for Bio-Preparedness, Chapel Hill, NC, USA; ${ }^{2}$ University of North Carolina, Chapel Hill, NC, USA; ${ }^{3}$ North

Carolina State University, Raleigh, NC, USA; ${ }^{4}$ SAS Institute, Cary, NC, USA

\section{Objective}

Demonstrate the functionality of the National Collaborative for Bio-Preparedness system.

\section{Introduction}

The National Collaborative for Bio-Preparedness (NCB-Prepared) was established in 2010 to create a biosurveillance resource to enhance situational awareness and emergency preparedness. This jointinstitutional effort has drawn on expertise from the University of North Carolina- Chapel Hill, North Carolina State University, and SAS Institute, leveraging North Carolina's role as a leader in syndromic surveillance, technology development and health data standards. As an unprecedented public/private alliance, they bring the flexibility of the private sector to support the public sector. The project has developed a functioning prototype system for multiple states that will be scaled and made more robust for national adoption.

\section{Methods}

NCB-Prepared recognizes that the capability of any biosurveillance system is a function of the data is analyzes. NCB-Prepared is designed to provide information services that analyze and integrate national data across a variety of domains, such as human clinical, veterinary and physical data. In addition to this one-health approach to surveillance, a primary objective of NCB-Prepared is to gather data that is closer in time to the event of interest. NCB-Prepared has validated the usefulness of North Carolina emergency medical services data for the purposes of biosurveillance of both acute outbreaks and seasonal epidemics (1).

A unique model of user-driven valuing of data-providing value back to the provider in their terms-motivates collaboration from potential data providers, along with timely and complete data. NCBPrepared approaches potential data providers, partners and users with the proposition that enhanced data quality and analysis is valuable to them individually and that an integrated information system can be valuable to all. With the onboarding of new data sources, NCB-Prepared implements a formal process of data discovery and integration. The goal of this process is three-fold: 1) to develop recommendations to enhance data quality going forward, 2) to integrate information across data sources, and 3) to develop novel analytic techniques for detecting health threats. NCB-Prepared is committed to both utilizing standard methods for event detection and to developing innovative analytics for biosurveillance such as the Text Analytics and Proportional charts method (TAP). The sophisticated analytic functionality of the system, including improved time to detection, query reporting, alert detection, forecasting and predictive modeling, can be attributed to collaboration between analysts from private industry, academia and public health.

NCB-Prepared followed the formal software development process known as agile development to create the user interface of the system. This method is based on iterative cycles wherein requirements evolve from regular sessions between user groups and developers. The result of agile development and collaborative relationships is a system which visualizes signals and diverse data sources across time and place while providing information services across all levels of users and stakeholders.

\section{Conclusions}

Lessons Learned:

1. Understand the functionality of new biosurveillance system, NCB-Prepared

2. Identify the benefit of creating collaborative relationships with data providers and users

3. Appreciate the value of a public/private partnership for biosurveillance and bio-preparedness

\section{Keywords}

Biosurveillance; Analytics; Preparedness; Emergency

\section{References}

Cairns C., Potenziani D., Hoit M., Jenkins C., Edgemon S. Novel approach to statewide biosurveillance using emergency medical services (EMS) information. Emerg Health Threats J 2011; 4, DOI: 4: 10.3402/ehtj.v4i0.11183 (abstract).

\section{* Meredith Arasaratnam}

E-mail: arasarat@med.unc.edu 